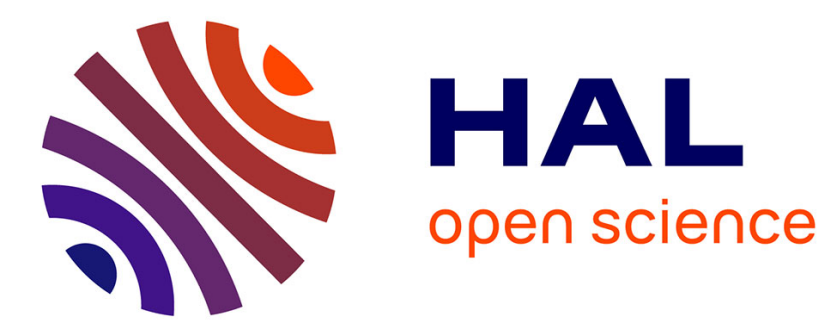

\title{
How to well pose a magnetization identification problem
}

Olivier Chadebec, Jean-Louis Coulomb, Gilles Cauffet, Jean-Paul Bongiraud

\section{To cite this version:}

Olivier Chadebec, Jean-Louis Coulomb, Gilles Cauffet, Jean-Paul Bongiraud. How to well pose a magnetization identification problem. IEEE Transactions on Magnetics, 2003, 39 (3), pp.1634-1637. 10.1109/TMAG.2003.810429 . hal-00289978

\section{HAL Id: hal-00289978 https://hal.science/hal-00289978}

Submitted on 24 Jun 2008

HAL is a multi-disciplinary open access archive for the deposit and dissemination of scientific research documents, whether they are published or not. The documents may come from teaching and research institutions in France or abroad, or from public or private research centers.
L'archive ouverte pluridisciplinaire HAL, est destinée au dépôt et à la diffusion de documents scientifiques de niveau recherche, publiés ou non, émanant des établissements d'enseignement et de recherche français ou étrangers, des laboratoires publics ou privés. 


\title{
How to Well Pose a Magnetization Identification Problem
}

\author{
Olivier Chadebec, Jean-Louis Coulomb, Gilles Cauffet, and Jean-Paul Bongiraud
}

\begin{abstract}
This paper presents an original approach for determining the unknown magnetization of a ferromagnetic shell. Magnetic measurements using sensors close from the device under test are used to rebuild distributions located on the shell. These distributions are representative of the magnetization and tangential moments or charges can be used. This identification problem is a particular case of an inverse problem and is generally ill posed. Instead of using classical mathematical tools to solve such a problem, we preferred to change it in a better posed one by adding our physical knowledge of the problem. All our results have been validated on a mockup with real measurements.
\end{abstract}

Index Terms-Charges identification, inverse problems, magnetization identification, magnetostatic, thin shell.

\section{INTRODUCTION}

$\mathbf{M}$ OST SHIPS' hulls are built with ferromagnetic materials (steel, etc.). A ship, placed in the earth's magnetic field, then gets a magnetization and creates a local anomaly of it. For military vessels, this anomaly can lead to their destruction by magnetic mines or their localization (submarine). The goal of this work is to develop a tool that allows the ship to predict its own magnetization and then its own magnetic risk.

A hull's magnetization has two kinds of components. The first one is the induced magnetization and is due to the reaction of the material when it is placed in an inductor field $\mathbf{H}_{\mathbf{0}}$ (i.e., earth's magnetic field). The calculus of this magnetization is now well-known and easy to compute [1], [2]. The second magnetization is the remanent one and depends on the magnetic history of the device (combination of mechanical and thermal constraints, magnetostriction, etc.). Because we have no idea of this magnetic history, a deterministic calculus is not possible. It is then necessary to use static magnetic measurements to determine it. In our application, sensors are necessarily placed onboard the ship. The goal of our work is, from these measurements, to reconstruct a model of the shell's magnetization. Once it is obtained, it will be easy to calculate the field outboard, where sensors cannot be placed.

\section{Two Kinds OF Distributions}

We consider a ferromagnetic shell placed in an external inductor field $\mathbf{H}_{\mathbf{0}}$. This device creates a local perturbation of the

Manuscript received June 18, 2002. This work was supported by the French Navy (DGA-GESMA).

O. Chadebec and J.-L. Coulomb are with the Laboratoire d'Electrotechnique de Grenoble, LEG/ENSIEG, Saint Martin d'Hères 38402, France (e-mail: chadebec@leg.ensieg.inpg.fr).

G. Cauffet and J.-P. Bongiraud are with the Laboratoire du Magnétisme du Navire, LMN/ENSIEG, Saint Martin d'Hères 38402, France.

Digital Object Identifier 10.1109/TMAG.2003.810429 field $\mathbf{H}_{\text {red }}$ which can then be expressed in term of reduced potential $\varphi_{\text {red }}$

$$
\mathbf{H}=\mathbf{H}_{\mathbf{0}}+\mathbf{H}_{\mathrm{red}}=\mathbf{H}_{\mathbf{0}}-\operatorname{grad} \varphi_{\mathrm{red}} .
$$

\section{A. Moments Distribution}

The hull has a magnetization $\mathbf{M}$ which is equal to the sum of the induced magnetization $\mathbf{M}^{\text {ind }}$ and the remanent one $\mathbf{M}^{\text {rem }}$

$$
\mathbf{M}=\mathbf{M}^{\text {ind }}+\mathbf{M}^{\mathrm{rem}} .
$$

If the thickness $e$ of the sheet is small and relative permeability $\mu_{r}$ high, we can consider that induced magnetization $\mathbf{M}^{\text {ind }}$ is parallel to the shell and constant through it [1]. We assume that it is the case for the remanent magnetization $\mathbf{M}^{\mathrm{rem}}$ too. We can then model the plate by a median surface $S$, where a distribution of the tangential magnetization $\mathbf{M}$ is located. It is directly linked to the reduced field (i.e., the field created by the shell) in the air region by the following [3]:

$$
\mathbf{H}_{\mathrm{red}}=\frac{e}{4 \pi} \iint_{S} \frac{\left(3(\mathbf{M}, \mathbf{r}) \mathbf{r}-\mathbf{r}^{2} \mathbf{M}\right.}{r^{5}} d S
$$

where $r$ is the vector between a point of the air region and the integration point of the median surface $\mathrm{S}$.

\section{B. Charges Distribution}

If we consider that

$$
\mathbf{B}=\mu_{0}(\mathbf{M}+\mathbf{H})
$$

and

$$
\operatorname{div} \mathbf{B}=0
$$

we obtain

$$
\mu_{0} \operatorname{div}\left(\mathbf{M}+\mathbf{H}_{0}-\operatorname{grad} \varphi_{\text {red }}\right)=0
$$

as $\operatorname{div} \mathbf{H}_{\mathbf{0}}=0$

$$
\Delta \varphi_{\text {red }}=\operatorname{div} \mathbf{M} .
$$

Equation (7) is equivalent to Poisson's equation; the magnetization of the shell can then be considered as equivalent to a distribution of charges located on $\mathrm{S}$. Thus, the relation between this distribution and the tangential magnetization is

$$
e \operatorname{div}_{S} \mathbf{M}=\rho=\rho^{\text {ind }}+\rho^{\text {rem }}
$$


where $\operatorname{div}_{S}$ is the standard divergence operator applied only on the tangential components of S. Field perturbation can then be expressed by

$$
\mathbf{H}_{\mathrm{red}}=\frac{e}{4 \pi} \iint_{S} \rho \frac{\mathbf{r}}{r^{3}} d S
$$

We notice that the charge distribution $\rho$ is a mathematical abstraction but represents the flux of induction entering the shell form the air region [4]. The advantage of (3) and (9) is their validity everywhere in the air region (i.e., outboard and onboard the device).

\section{INVERSE PROBLEM}

The aim of our work is, starting from measurements of the field in the air, to determine these moments or charges distributions. We have written (3) and (9) which can link a measured field (in fact the reduced measured field) to theses distributions. The goal of our work is to invert them. We mesh $S$ into $N$ surface elements and $M$ nodes. It remains to find shapes function with respect to each distribution and its variation on the elements.

\section{A. Shape's Functions}

1) Moments Shapes Functions: We can assume that variations of magnetization on each element are not significant. It seems sufficient to consider that magnetization is constant on each element (zeroth-order approximation). We notice that (3) is analytically convergent but its numerical integration can lead to divergence ( $1 / r^{3}$ field variation at the proximity of the shell). Moreover, we would like to place sensors close to the shell (and the sources), it is then more efficient to use a classical divergence theorem

$\mathbf{H}_{\mathrm{red}}=\frac{1}{4 \pi} \iiint_{V} \frac{\left(3(\mathbf{M} \cdot \mathbf{r}) \mathbf{r}-r^{2} \mathbf{M}\right.}{r^{5}} d V=-\frac{1}{4 \pi} \iint_{S} \mathbf{M} \cdot \mathbf{n} \frac{\mathbf{r}}{r^{3}} d S$

where $V$ is a volume with a constant magnetization, delimited by $S$ and with an external normal $\mathbf{n}$. By combining (3) and (10) reduced to a surface element, we obtain

$$
\mathbf{B}_{\text {mes }}=\mu_{0}\left(\mathbf{H}_{\mathbf{0}}-\frac{e}{4 \pi} \sum_{i}^{N} \int_{L_{i}}\left(\mathbf{M}_{\mathbf{i}}^{\text {ind }}+\mathbf{M}_{\mathbf{i}}^{\mathrm{rem}}\right) \cdot \mathbf{n}_{\mathbf{i}} \frac{\mathbf{r}}{r^{3}} d L_{i}\right)
$$

where $\mathbf{B}_{\text {mes }}$ is the measured field, $L_{i}$ is the edge of element $\mathrm{i}$, and $\mathbf{n}_{\mathbf{i}}$ the external normal of $S_{i}$ tangential to $\mathrm{S}$. Integrations are now realized on edges of the mesh. We can then notice that a constant magnetization $\mathbf{M}_{\mathbf{i}}$ on each element $i$ is strictly equivalent to a constant line distribution of charge $\mathbf{M}_{\mathbf{i}} \cdot \mathbf{n}_{i}$ located of the edge of element i. We have then found a relation between the measured field and a constant distribution of magnetization per element. This relation has been written in terms of constant charge distribution per edge to limit the singularity $\left(1 / r^{2} \sin\right.$ gularity near the shell). We notice that unknowns of (11) are still $\mathbf{M}_{\mathbf{i}}$.

2) Charges Shape's Functions: We remember that a charge distribution represents the flux of induction entering the shell form the outside and the inside air regions. It then seems natural

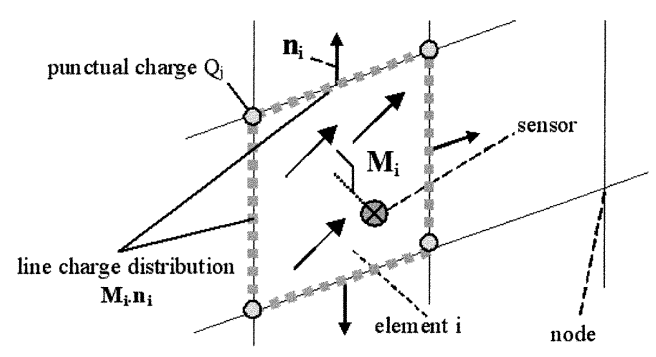

Fig. 1. Sensor location in front of element's barycenter with line uniform charge distributions and punctual distributions.

to locate charges where this flux could have a high value, in particular, on edges or corners of the device. So, we have decided to locate punctual charges $Q$ on each node of the mesh. Then, we obtain the following:

$$
\mathbf{B}_{\text {mes }}=\mu_{0}\left(\mathbf{H}_{\mathbf{0}}-\frac{e}{4 \pi} \sum_{j}^{M}\left(Q_{j}^{\text {ind }}+Q_{j}^{\mathrm{rem}}\right) \frac{\mathbf{r}}{r^{3}} .\right.
$$

Equation (12) is a less good approximation of the distribution than (11) (line integration of charges against punctual charges). We will see the reasons for this choice in a following section.

\section{B. Sensors Location}

We have seen that (11) and (12) presents a $1 / r^{2}$ numerical singularity near the shell. It is then necessary to place sensors far from these distributions. Our choice is to locate them directly in front of the barycenter of each element. Integration would then be done with quite good accuracy (see Fig. 1).

\section{Writing of the Inverse Linear System}

We now place $K$ magnetic triaxial sensors inside the hull and close to it. We can then build a system where the unknowns are the sum of remanent and induced moments or charges.

$$
\mathbf{A} \mathbf{x}=\mathbf{A}\left(\mathbf{x}^{\text {ind }}+\mathbf{x}^{\mathrm{rem}}\right)=\mathbf{b}
$$

where $\mathbf{A}$ is a $(3 K \times 2 N)$ matrix for the moments case and a $(3 K \times M)$ matrix for the charges one. The goal is now to find $\mathbf{x}$ so to solve an inverse problem. $x=\mathbf{M}$ corresponds to moments identification and $x=Q$ to the charges one.

\section{Rank-Deficient and Ill-Posed Problems}

Most inverse problems are said to be ill posed and their solutions are not unique. It is the case for our application too. Two different causes could lead to an ill-posed problem [5].

1) Rank-Deficient Problem: We consider that we have few sensors and that they are far from each other. We then have fewer equations than unknowns and (13) is purely underdetermined. Indeed, every linear combination of vectors from the kernel of $\mathbf{A}$ is not observable and an infinite number of solutions $\mathbf{x}$ exactly fit the data. The problem is then said to be rank deficient. An example of sensors location which leads to such a system is shown in Fig. 2.

To solve these kinds of systems, single-value decomposition (SVD) is usually used. The SVD then returns the solution $\mathbf{x}$ which fits the measurements and has the minimal norm. We have tried to solve (12) and (13) for rank-deficient systems. Our experience can be resumed by the two following points. 


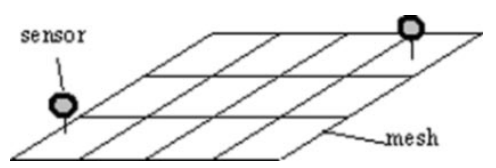

Fig. 2. Typical case of rank-deficient problem $(N=9,6$ equations-18 unknowns for moments identification)

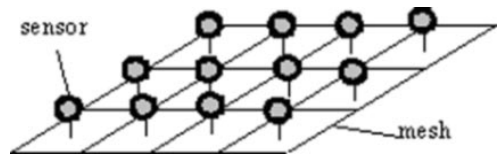

Fig. 3. Typical case of problem with bad condition number $(N=9,27$ equations-18 unknowns for moments identification).

- Charges identification: The solution may be acceptable. Indeed, SVD naturally minimizes the flux entering the shell. However, we think that inversions on geometries where the flux has high variations can fail.

- Moments identification: The solution with the minimal norm is not acceptable. In particular, elements far from sensor get a magnetization that is too small.

2) Problem With Poor Condition Number: We now consider that we have lots of sensors close to each other. Several different distributions of sources can create a field very close to the measured one. It is then very difficult to favor a solution, the measurements being noisy. In that sense, the solution is not unique either. This fact is due to the presence of quasicontradictory equations in the system. The resolution of (13) leads then to an unacceptable divergent solution. The parallelism between equations can be evaluated by the condition number of matrix A (ratio between the highest and smallest single values of matrix $\mathbf{A}$ ). If the condition number is close to one, equations are strongly independent; on the contrary, a high condition number leads to amplification of measurement inaccuracies and to an unstable solution. Let us note that a high number of measurements increases the phenomena, by increasing the number of equations and then increasing the condition number too, especially for the case of more equations than unknowns (Fig. 3).

The inverse problem needs then to be regularized. The most used regularization method has been developed by Tikhonov [6] and consists of solving the following by SVD:

$$
(\mathbf{A}+\alpha \mathbf{L})\left(\mathbf{x}^{\text {ind }}+\mathbf{x}^{\text {rem }}\right)=\mathbf{b}
$$

where $\alpha$ is a weighted parameter. If $\mathbf{L}$ equal $\mathbf{I}_{\mathbf{d}}$ (i.e., the identity matrix), the method tries to fit the distribution to the magnetic measurements by keeping a minimal norm for $\mathbf{x}$ (zeroth-order regularization) and avoiding then divergence of the solution. $\mathbf{L}$ can also be chosen as the derivative operator, the solution returned is then the most regular.

- Charges identification: Zeroth-order regularization succeeds (flux minimization) but the choice of the weighted parameter still remains a difficulty.

- Moments identification: Inversion fails. Indeed, as we already said for magnetization, a solution with a small norm is not satisfactory. It is then better to find the most regular solution. $\mathbf{L}$ must be chosen as the derivative matrix (first-order regularization). However, this approach is difficult to apply to three-dimensional problems. The geometrical complexity of our device and our vectors basis (for moments distribution) makes matrix $\mathbf{L}$ very difficult to compute. Moreover, the choice of the weighted parameter remains a difficulty.

We notice that a magnetization identification problem can have both difficulties (for example, if few sensors are close to each other).

\section{STRATEGY FOR SOLVING IDENTIFICATION MAGNETIZATION PROBLEM}

Classical approaches favor applications with lots of measurements and then use regularization theory [3]. However, we decide not to follow this way. There are several reasons: first, devices with lots of sensors are expensive and difficult to manage. Then the choice of the regularization parameter is difficult and measurement dependant. Finally, we would like to solve the moments inversion to have a physical representation of the magnetization.

We decided then to use only few sensors and then to solve a severely rank-deficient system. The sensor location is chosen to ensure good condition number. In particular, they are far from each other to obtain strong decoupled equations. The kernel of the system has then a high dimension, and a high number of distributions fit measurements. To obtain a pertinent solution, the new solution that we propose is to reduce its dimension. It can be done by adding some other equations. In fact, our application is really specific and a fundamental aspect of the problem can be taken into account: The shell channels the flux of induction by following Maxwell's laws. We consider a model for the ferromagnetic material which takes into account remanent magnetization

$$
\mathbf{B}=\mu \mathbf{H}+\mathbf{B}^{\text {rem }}
$$

where $\mu$ is reversible linear permeability. We also have

$$
\mathbf{B}=\mu_{0}\left(\mathbf{H}+\mathbf{M}^{\text {ind }}+\mathbf{M}^{\text {rem }}\right)
$$

and then, by combining (15) and (16)

$$
\mathbf{M}^{\text {ind }}=\left(\mu_{r}-1\right) \mathbf{H}
$$

where $\mu_{r}$ is the reduced reversible permeability of the material. With (1), an equation similar to (11) and (17), we get with a collocation method at the barycenter on element $i$ of the mesh [7]

$$
\begin{gathered}
\mathbf{M}_{\mathbf{j}}^{\text {ind }}=\left(\mu_{r}-1\right)\left(\mathbf{H}_{\mathbf{0}}-\frac{e}{4 \pi} \sum_{i}^{N} \int_{L_{i}} \mathbf{M}_{\mathbf{i}}^{\text {ind }} \cdot \mathbf{n}_{\mathbf{i}} \frac{\mathbf{r}}{r^{3}} d L_{i}+\right. \\
\left.-\frac{e}{4 \pi} \sum_{i}^{N} \int_{L_{i}} \mathbf{M}_{\mathbf{i}}^{\text {rem }} \cdot \mathbf{n}_{\mathbf{i}} \frac{\mathbf{r}}{r^{3}} d L_{i}\right) .
\end{gathered}
$$

A similar approach can be applied with the charges approach and a flux balance equilibrium equation [2]

$$
\begin{aligned}
Q_{i}^{\text {ind }}=\left(\mu_{r}-1\right) & \left(e \int_{L_{j}} \mathbf{H}_{\mathbf{0}_{\mathbf{S}}} \cdot \mathbf{n}_{j} d L+e \sum_{j}^{M} Q_{j}^{\text {ind }} \int_{L_{j}} \frac{\mathbf{r}}{r^{3}} \mathbf{n}_{\mathbf{j}} d L\right. \\
& \left.+e \sum_{j}^{M} Q_{j}^{\mathrm{rem}} \int_{L_{j}} \frac{\mathbf{r}}{r^{3}} \mathbf{n}_{\mathbf{j}} d L\right)
\end{aligned}
$$




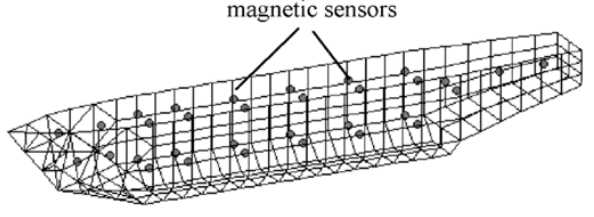

Fig. 4. Mesh and sensors location (28 sensors, 271 elements, 273 nodes).

By writing (18) or (19) on each element of the mesh, we obtain two new square systems valid inside the shell. We notice that these systems do not deal with measurements but only with the inductor field and the relative permeability

$$
\left(\mathbf{C}+\mathbf{I}_{\mathbf{d}}\right)\left(\mathbf{x}^{\text {ind }}+\mathbf{x}^{\text {rem }}\right)=\mathbf{d}
$$

where $\mathbf{I}_{\mathbf{d}}$ is the identity matrix.

Other considerations can improve our knowledge of the problem and leads to one equation more. We remember that a distribution of charge represents the flux of induction entering the shell; according to the physic the sum of these charges must be null. We then get two new equations for moments

$$
\sum_{\mathbf{i}}^{\mathbf{N}} \int_{\mathbf{L}_{\mathbf{i}}}\left(\mathbf{M}_{\mathbf{i}}^{\text {ind }}+\mathbf{M}_{\mathbf{i}}^{\text {rem }}\right) \cdot \mathbf{n}_{\mathbf{i}} d L_{i}=0
$$

and the punctual charges problem

$$
\sum_{j}^{M}\left(Q_{j}^{\text {ind }}+Q_{j}^{\text {rem }}\right)=0 .
$$

It remains now to solve a global system for the two problems

$$
\left[\begin{array}{cc}
A & A \\
\hline C+I_{d} & C \\
\hline s & s
\end{array}\right]\left[\begin{array}{l}
x^{\mathrm{ind}} \\
x^{\mathrm{rem}}
\end{array}\right]=\left[\begin{array}{l}
b \\
d \\
0
\end{array}\right] .
$$

The solution returned by these resolutions is now the distribution that fits the measurements and moreover that takes into account the behavior of the material (according to Maxwell's laws). Our knowledge of the problem is now much better than before. Moreover, (18) and (19) have both good condition numbers, therefore, it will also be the case for global systems, so it is then useless to regularize it. Systems are solved with a simple SVD.

\section{NUMERICAL APPLICATION}

Our two approaches have been validated on a real mockup representative of a real ship (4.60 m long). 28 fluxgate magnetometers have been placed onboard (Fig. 4). The thickness, the relative permeability of the shell, and the inductor field are well-known. The shell is meshed into 271 surface elements, in both cases, systems (13) obtained are then severely rank deficient. It is then indispensable to solve system (23) to get a pertinent solution. Distributions obtained are shown in Figs. 5 and 6.

Once solutions have been obtained, is it easy to compute the field everywhere in the air region, with the help of (11) and (12). This field, predicted outboard, has been compared to a measured one. Results for two kinds of distributions present a difference fewer than $5 \%$ of differences [7]. We can then conclude that the identification has been done with good accuracy.

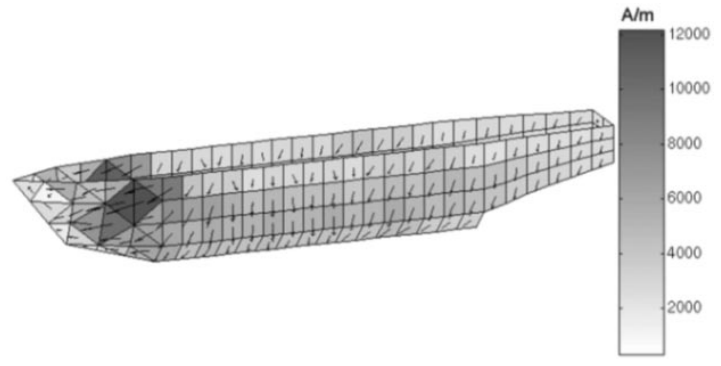

Fig. 5. Moments distribution obtained by identification with 28 sensors on a real mockup. Measurements system: 96 equations for 1024 unknowns. Internal system: 543 equations added.
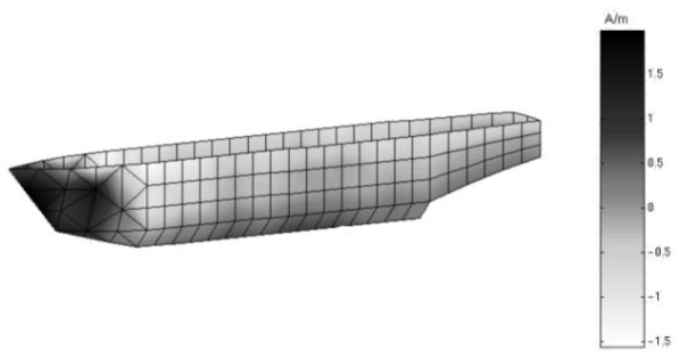

Fig. 6. Charge distribution obtained by identification with 28 sensors on a real mockup. Measurements systems: 96 equations for 546 unknowns. Internal system: 274 equations added.

\section{CONCLUSION}

In this paper, we have explained a methodology to change an ill-posed problem to a better posed one. This approach consists of favoring the knowledge of the behavior of sources to the detriment of the measurement. Instead of using numerical tools to solve an ill-posed problem, we have preferred to pose a better one. This approach has many advantages. First no empirical parameters are needed. Then fewer sensors are used and the approach seems to be very stable by ensuring a coherent distribution of sources. According to our experience the punctual charge distribution identification seems to be the most reliable but does not lead to a representation of the magnetization in terms of moments.

\section{REFERENCES}

[1] X. Brunotte and G. Meunier, "Line element for efficient computation of the magnetic field created by thin iron plates," IEEE Trans. Magn., vol. 26, pp. 2196-2198, Sept. 1990.

[2] A. Vishnevski, I. Krasnov, and A. Lapokov, "Calculation of static magnetization for thin-walled constructions by boundary element method,' IEEE Trans. Magn., vol. 29, pp. 2152-2155, Sept. 1993.

[3] H. Igarashi, T. Honma, and A. Kost, "Inverse inference of magnetization distribution in cylindrical permanent magnets," IEEE Trans. Magn., vol. 36, pp. 1168-1171, July 2000.

[4] O. Chadebec, J.-L. Coulomb, V. Leconte, J. P. Bongiraud, and G. Cauffet, "Modeling of static magnetic anomaly created by iron plates," IEEE Trans. Magn., vol. 36, pp. 667-671, Oct. 2000.

[5] C. Hansen, Rank Deficient and Discrete Ill-Posed Problems. Philadelphia, PA: SIAM, 1998.

[6] A. N. Tikhonov and V. Y. Arsenine, Solution of Ill-Posed Problem. Washington, DC: Wiston/Wiley, 1977.

[7] O. Chadebec, J.-L. Coulomb, J.-P. Bongiraud, G. Cauffet, and P. Le Thiec, "Recent improvements for solving inverse magnetostatic problem applied to thin shells," IEEE Trans. Magn., vol. 38, pp. 1005-1008, Mar. 2002. 